\title{
Molecular Cloning and Characterization of Soybean Cinnamoyl CoA Reductase Induced by Abiotic Stresses
}

\author{
Hyun-Ah So ${ }^{1, \dagger}$, Eunsook Chung ${ }^{1, \uparrow}$, Chang-Woo Cho ${ }^{1}$, Kee-Young Kim ${ }^{2}$ and Jai-Heon Lee ${ }^{1 *}$ \\ ${ }^{1}$ BK21 Center for Silver-Bio Industrialization, Dong-A University, Busan 604-714, Korea \\ ${ }^{2}$ National Academy of Agricultural Science, RDA, Suwon 441-707, Korea \\ (Received on August 10, 2010; Accepted on October 26, 2010)
}

\begin{abstract}
Suppression subtractive hybridization was used to isolate wound-induced genes from soybean. One of the wound-induced genes, gmwi143 designated as GmCCR, showed high homology with genes encoding cinnamoylCoA reductase (CCR; EC 1.2.1.44). Deduced amino acid sequences encoded by GmCCR showed the highest identity (77\%) with those of Acacia CCR. There are 2 CCR genes highly homologous to $G m C C R$ in soybean genome based on Phytozome DB analysis. RNA expression of $G m C C R$ was specifically induced by local and systemic wounding, drought, high salinity or by ultraviolet stress. Our study suggests that $G m C C R$ may be involved in resistance mechanism during abiotic stresses in plants.
\end{abstract}

Keywords : cinnamoyl-coA reductase, lignin, soybean, wounding, UV

Lignin, a major component of secondary cell walls, is deposited mainly in the vascular tissues during plant development and environmental signals (Boerjan et al., 2003). Lignins contribute to major functions in plants, such as stem rigidity, water transport in xylem and are also involved in defense reactions against wounding, predators or pathogens (Vance et al., 1980). It has been reported that lignin biosynthesis is regulated by developmental signal in the vascular tissues and by defense responses in plant (Lauvergeat et al., 2001).

The biosynthesis of lignins begins with the common phenylpropanoid pathway, starting from phenylalanine and leading to cinnamoyl CoAs which are the common precursors of a wide range of phenolic compounds. These CoA esters subsequently are changed to monolignols via two reductive steps catalyzed by cinnamoyl CoA:NADP oxidoreductase (CCR, EC 1.2.1.44) and cinnamyl alcohol dehydrogenase (CAD, EC 1.1.1.195). Lignins result from the oxidative polymerization of monolignols.

\footnotetext{
*Corresponding author.

Phone) +82-51-200-7592, FAX) +82-51-200-7505

E-mail)jhnlee@dau.ac.kr.

'Equally contributed
}

Several genes encoding CCR have been shown to be transcriptionally induced by developing xylem tissues or by biotic stress (Kim et al., 2000; Lacombe et al., 1997; Lauvergeat et al., 2001). Lacombe et al. (1997) showed that Eucalyptus $C C R$ transcript was expressed in lignified organs such as leaves, stems and roots. By in situ hybridization technique, EuCCR was strongly expressed in the differentiating xylem zone but was not present in the secondary phloem fibers or in the young periderm (Lacombe et al., 1997). Rice OsCCR1 RNA expression was induced in suspension cell cultures treated with sphingolipid elicitor purified from rice blast Magnaporthe grisea (Kawasaki et al., 2006). Previously, soybean chip analysis showed that RNA expression of $C C R$ invovled in phenylpropanoid pathway were induced in the syncytia laser microdissected soybean roots infected with soybean cyst nematode (Klink et al., 2009).

There are 11 putative CCR homologues sharing homology identities raging from 82.8 to $32.8 \%$ in Arabidopsis genome (Costa et al., 2003). Arabidopsis AtCCR1 transcript was expressed in the stem and floral tissues (Lauvergeat et al., 2001) and the irregular xylem 4 mutant (irx4) defective in the AtCCR1 gene showed severely reduced lignin contents (50\%) compared to the wild type (Jones et al., 2001). Lauvergeat et al. (2001) suggested that AtCCR2 responsive to pathogen infection of Xanthomonas campestris pv. campestris may play a role in the formation of phenolic compounds associated with HR in Arabidopsis (Lauvergeat et al., 2001).

In the present study, we have isolated the full-length cDNA of $G m C C R$ encoding CCR-like protein regulated by abiotic stresses. We propose that the GmCCR is involved in defense responses during environmental stresses in plants.

\section{Materials and Methods}

Plant materials and treatments. Soybean plants (Glycine max cv. 'Sinpaldal 2') were grown in a growth chamber with $16 \mathrm{~h}$ /day light supply $(22,000 \mathrm{lux})$ at $28^{\circ} \mathrm{C}$ for $3-4$ weeks. Soybean leaves from the whole plant were cut with scissors as a wounding stress. The wounded leaves were 
sampled for the local wounding and the normal leaves far from the wounded leaves were for the systemic wounding sample at the indicated time. For drought stress, whole plants were removed from the soil and dehydrated at $28^{\circ} \mathrm{C}$ upto $24 \mathrm{~h}$. To induce high salinity stress, plants were soaked in a solution of $\mathrm{NaCl}(250 \mathrm{mM})$ at $28^{\circ} \mathrm{C}$ for $24 \mathrm{~h}$. For the UV-B treatment, whole plants were irradiated with UV light at the indicated time. Plant samples were immediately frozen in liquid nitrogen, and stored at $-75^{\circ} \mathrm{C}$.

Suppression subtractive hybridization (SSH). SSH was performed using PCR-Select cDNA Subtraction kit (Clontech Laboratories Inc., USA) as described by the manufacturer. For PCR-based cDNA subtraction method, all of the procedure was carried out according to the manufacture instructions. The cDNA synthesized from extracted mRNA at untreated leaves was used for driver. Tester was prepared at cDNA from combined mRNA that was extracted wounding treated leaves. First and second strand cDNA synthesis and blunt ending of DNA ends were carried out according to manufacture instructions (Clontech, USA). Product from the second PCR was inserted into pCR 2.1 vector using a TA Cloning Kit (Invitrogen, USA). Positively selected clones were sequenced (Genotech, Korea) and the sequences were analyzed using BLAST program by comparing with GenBank/EMBL database.

Rapid amplification of cDNA ends (RACE). Complementary DNA synthesis was carried out using reverse transcription kit (Promega). The full-length $G m C C R$ cDNA was obtained using SMART RACE cDNA amplification kit (Clontech) according to the manufacturer's instruction. Gene-specific 5' primer FSP1-GmCCR (5'-ATCGACGAAGTCGCCACCACGCG-3') and 3' primer RSP1-GmCCR (5'-CTTGAGAACAAGAAAGCAGCAGGAAGGCAC$3^{\prime}$ ) were used for RACE. The full-length cDNA of $G m C C R$ was PCR-amplified with a 5'-GmCCR primer (5'-CAAAGCAACCCTATGTCGAAGGTGG-3') and the 3'-GmCCR primer (5'-ACTCAGTCCCTCTGCAGCAG-3'). The resulting PCR product was cloned to pGEMT-Easy (Promega) for the sequencing.

Northern blot and Southern blot analysis. Total RNA sequences were isolated using RNA extraction Kit (Ambion). Total RNA (20 mg) was fractionated on a $1 \%$ formaldehyde agarose gel, and transferred to a nylon membrane. The GmCCR probe was PCR-amplified using a 5'-GmCCR primer (5'-CAAAGCAACCCTATGTCGAAGGTGG-3') and the 3'-GmCCR primer (5'-ACTCAGTCCCTCTGCAGCAG-3') covering the entire cDNA region of $G m C C R$ gene (966 bp). The probe was labeled by a random priming method using the Ladderman labeling kit (Takara).
The membrane was hybridized to $\mathrm{a}^{32} \mathrm{P}-\mathrm{dCTP}$ labeled probe using fragments encompassing GmCCR cDNA and washed, as described by Church and Gilbert (1984) prehybridization for $2 \mathrm{~h}$ at $42^{\circ} \mathrm{C}(0.5 \mathrm{M}$ sodium phosphate $\mathrm{pH} 7.0,1$ $\mathrm{mM}$ EDTA, $7 \%$ SDS), hybridization overnight at $42^{\circ} \mathrm{C}$, washes at room temperature ( $0.5 \times \mathrm{XSC}, 0.1 \% \mathrm{SDS})$ and then $65^{\circ} \mathrm{C}(0.1 \mathrm{X} \mathrm{SSC}, 0.1 \%$ SDS $)$. The membrane was exposed to X-ray film (Kodak) for detection of signals.

Genomic DNA was extracted from the leaf of soybean (Glycine max. cv Sinpaldal 2) as described by Shure et al. (Shure et al., 1983). Genomic DNA from the leaf of soybean was digested with $X b a \mathrm{I}, E c o$ RI and HindIII and then electrophoresed through $1.0 \%$ agarose gel. The fractioned agarose gel was denatured with a denaturation solution $(0.5$ $\mathrm{M} \mathrm{NaOH}, 1.5 \mathrm{M} \mathrm{NaCl}$ and then renatured in a neutralization solution ( $0.5 \mathrm{M}$ Tris- $\mathrm{HCl}, 1.5 \mathrm{M} \mathrm{NaCl}, \mathrm{pH} 7.5)$. The DNA was transferred onto a nylon membrane. To detect GmCCR, 966-bp DNA fragments covering the full-length cDNA of $G m C C R$ was used for probe labeling. Probe labeling, prehybridization, hybridization and washing were conducted as the same as Northern blot analysis.

\section{Results and Discussion}

Cloning and sequence analyses of a gene encoding cinnamoyl-CoA reductase from soybean. In this study, we report molecular characterization of $G m C C R$ cDNA from soybean obtained from SSH to isolate genes differentially expressed during wounding stress (Chung et al., 2007). One of wound-inducible cDNA encoding CCR family protein was designated as GmCCR ( $\underline{\text { Glycine }}$ max Cinnamoyl-CoA Reductase) (Fig. 1A). The full-length cDNA of $G m C C R$ induced by wounding stress was obtained by $5^{\prime}$ and 3' RACE using cDNA of soybean leaf with a pair of specific primer as described in materials and methods (Genbank number; ABY81885). The full-length cDNA sequence of $G m C C R$ is composed of an open reading frame (966 bp) encoding a protein of 321 amino acids (MW, 35.2 $\mathrm{kDa}$; pI 5.66).

A multiple sequence alignment of the deduced protein sequence of $G m C C R$ gene with other CCR protein sequences is shown in Fig. 1A. The GmCCR showed $77 \%$ protein sequence identity to CCR of Acacia (AAY86360), 66\% to that of A. thaliana (AtCCR12), 67\% to that of grapevine (Fig. 1A). Multiple alignment of GmCCR and other CCR proteins showed that in the $\mathrm{N}$-terminal region (pos. 7-27 aa) a highly conserved motif is found: VTG(A/G)(A/G)G(F/ Y)IAS(G/W)(L/M)VK(I/L/R)LL(D/E) (K/R)GY (Fig. 1A). This conserved sequence represents a dinucleotide binding domain of NADP $(\mathrm{H})$ in NADH-dependent reductases and dehydrogenases (Lacombe et al. 1997) (Fig. 1A). This suggests that GmCCR and other CCRs have the amino acids 
A

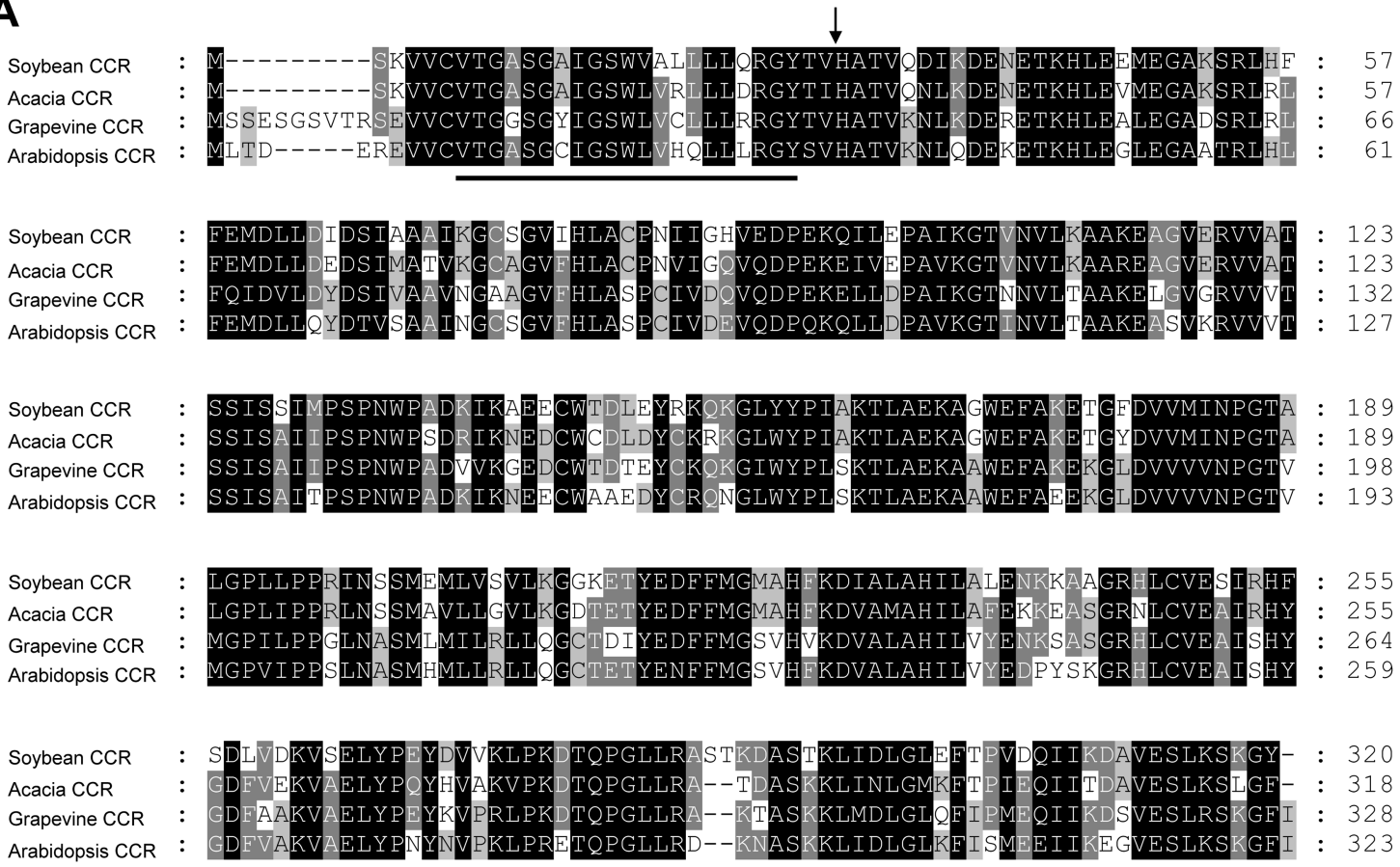

B

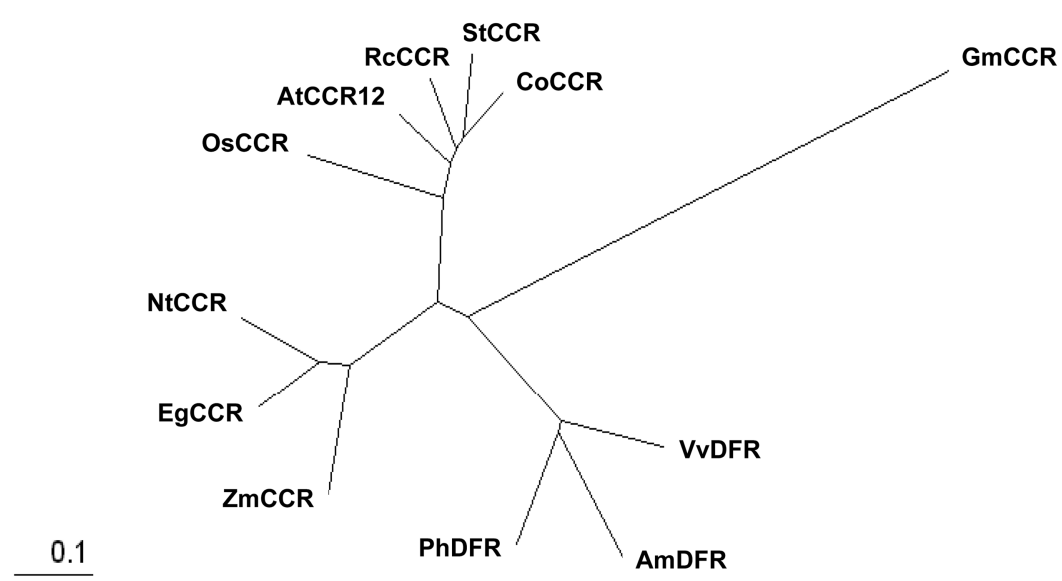

Fig. 1. Multiple alignment and phylogenetic tree of the deduced amino acid sequences of GmCCR with CCRs from other plant species. (A) Alignments of sequences were performed using the Clustal W program on the EBI WWW molecular biology server. The N-terminal signal peptide predicted for vacuole targeting sequence is marked by arrow and the putative NAD (P) binding domain found in other CCRs is underlined. The Accession numbers of the sequences used in the comparison are: Acacia mangium x A. auriculiformis, AAY86360; Arabidopsis thaliana, AAO22571; Glycine max, ABY81885 (in this study); Vitis vinifera, CAO21401. (B) The tree was constructed using the Clustal method based on amino acid similarities of the full sequences. The Accession numbers of the sequences used in the comparison are: Arabidopsis thaliana, AAO22571 (AtCCR12); Glycine max, ABY81885 (GmCCR); Oryza sativa, NP 001051706 (OsCCR); Ricinus communis, EEF35783 (RcCCR); Camellia oleifera, ACQ41893 (CoCCR); Solanum tuberosum, ABA46761 (StCCR); Zea mays, X98083 (ZmCCR); Nicotiana tabacum, A47101 (NtCCR); Eucalyptus gunnii, X79566 (EgCCR); Petunia hybrida, X15537 (PhDFR); V. vinifera, X75964 (VvDFR); Antirrhinum majus, X15536 (AmDFR).

residues strictly conserved in the mammalian $3 \beta$-hydroxysteroid dehydrogenase/CCR superfamily (Lacombe et al. 1997) (Fig. 1A). However, there is no catalytic site of CCR, which is a conserved motif, NWYCY found in CCRs (Fig. 1A) (Lacombe et al., 2000; Lauvergeat et al., 2001).

A phylogenetic analysis of 12 different plant CCRs and
DFRs was carried out using the Clustal method (Fig. 1B). As seen in the evolutionary tree of amino acid sequences, GmCCR lies between CCR and DFR (Fig. 1B). Protein sequence identity of $\mathrm{GmCCR}$ ranges from $56 \%$ with $\mathrm{OsCCR}$ to $66 \%$ to AtCCR12 (Fig. 1B). Another CCR group such as Eucalyptus EgCCR shows lower identities between $40 \%$ 
and $46 \%$ with GmCCR (Fig. 1B). The CCR group more similar to GmCCR lacks the conserved catalytic domain NWYCY found in other functional CCRs with biochemical activities (Lacombe et al., 1997; Lauvergeat et al., 2001). The overall identities between GmCCR and dihydroflavonol4-reductase (DFR), the first specific enzyme of anthoccyanin biosynthesis proteins was much lower from $31 \%$ to $36 \%$ (Fig. 1B). It was found that CCR and DFRs, share a striking homology due to the presence of the conserved domain such as NADPH-dependent reductases (Lacombe et al., 1997).

Copy number and RNA expression of $G m C C R$. To estimate the copy number of GmCCR gene in the soybean genome, southern blot analysis was performed (Fig. 2). It was shown that there was one major band in EcoRI- or HindIII-lane but two bands in the XbaI-digested sample (Fig. 2). However, blast search reveals that there are two $C C R$ genes sharing high homology (100 and 95\%) and two genes having large difference with $G m C C R$ in soybean genome (Phytozome; www.phytozome.net) (Fig. 2). This may be natural because soybean genome is comprised of tetraploid $(2 \mathrm{n}=4 \times)$. It has been proven that AtCCR2 and EUCCR were present as single copy in Arabidopsis and Eucalyptus genome (Lacombe et al., 1997; Lauvergeat et al., 2001). Gene expression and the biochemical functions of each $C C R$ were shown to be different despite the annotated CCR. Therefore, Costa et al. (2003) proposed that it should remain to characterize gene expression and also their precise metabolic roles in vivo (Costa et al., 2003).

To investigate GmCCR RNA expression in soybean plants with abiotic stresses, Northern blot was carried out (Fig. 3). Since $G m C C R$ was isolated from wound-inducible

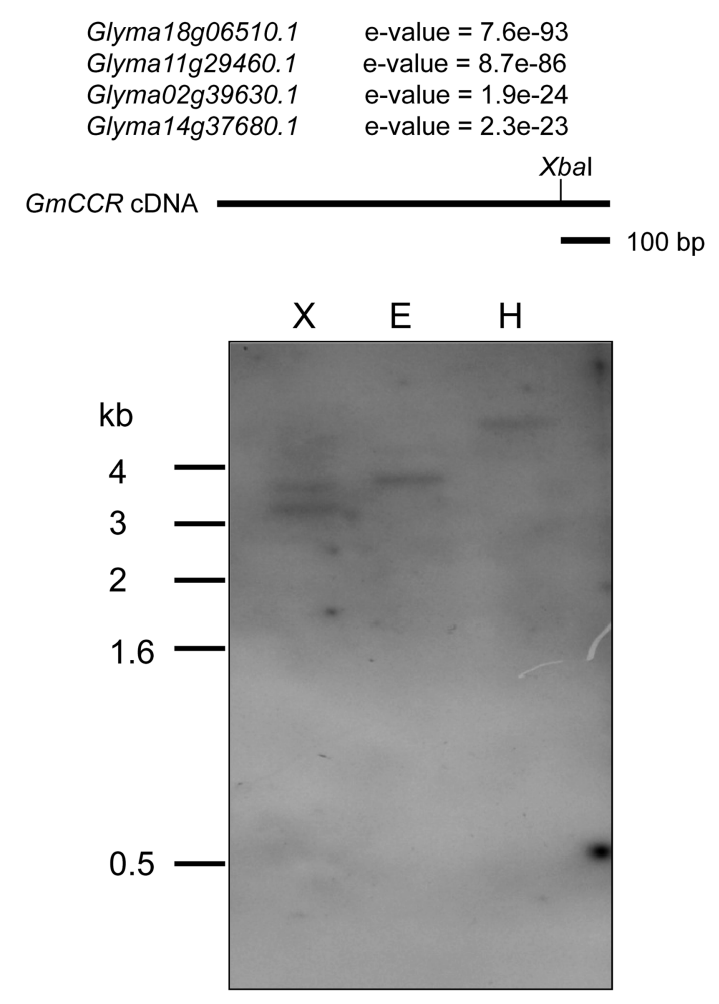

Fig. 2. Southern blot analysis of soybean genomic DNA for $G m C C R$. Twenty microgram of genomic DNA was digested with $X b a \mathrm{I}, E c o R \mathrm{I}$, or HindIII and loaded on each lane. PCR products corresponding to the full-length cDNA of $G m C C R$ was used for probe labeling.

cDNAs, GmCCR RNA expression was estimated in the wound-treated leaf tissues (Fig. 3A). GmCCR RNA level showed maximum after $6 \mathrm{~h}$ by wounding stress and its RNA level was reduced after that (Fig. 3A). GmCCR RNA
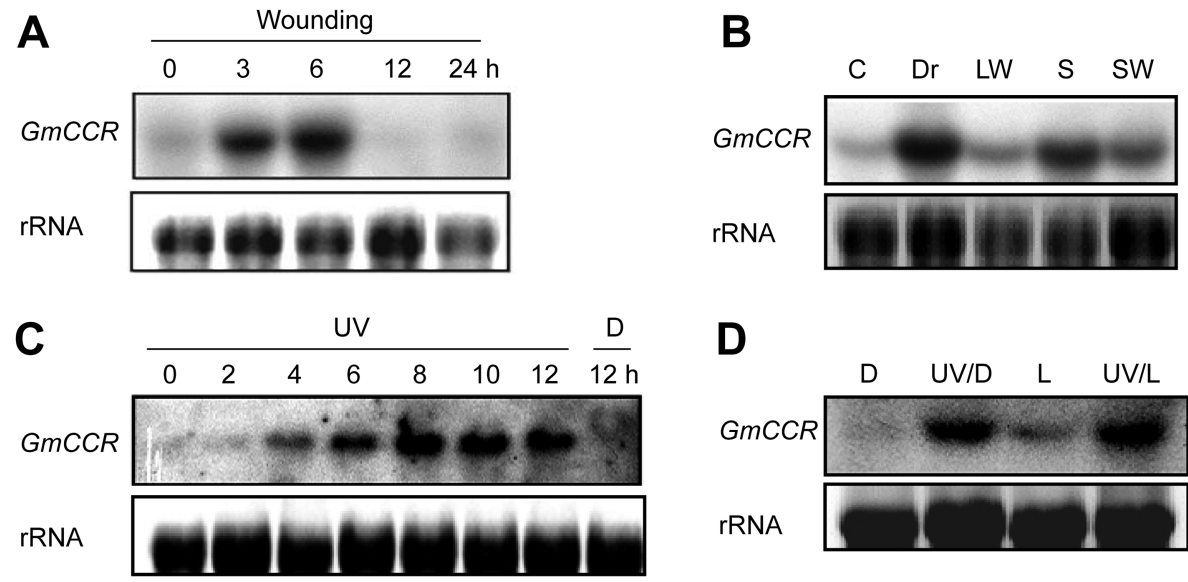

Fig. 3. Expression of $G m C C R$ gene by wounding, drought, high salinity or UV-B stresses. (A) Soybean leaves were wounded for 0 , 3, 6, 12 , or $24 \mathrm{~h}$. (B) Soybean plants were treated with drought (Dr), high salinity (S; $250 \mathrm{mM} \mathrm{NaCl}$ ) local wounding (LW) or systemic wounding (SW) for $24 \mathrm{~h}$. (C) Soybean seedlings grown under dark (D) or light (L) conditions, were exposed to UV for 8 h. (D) Soybean seedlings were exposed to UV upto $12 \mathrm{~h}$ under light conditions or to dark (D). Twenty microgram of total RNAs was loaded on each lane. RNA blot analysis was carried out as described in Materials and Methods. 
expression was monitored by other abiotic stresses such as drought, local and systemic wounding and high salinity $(\mathrm{NaCl})$ stresses (Fig. 3B). GmCCR RNA was strongly induced by drought or high salinity stress (Fig. 3B). Both local and systemic wounding stresses slightly induced GmCCR RNA expression (Fig. 3B).

UV-B radiation induces secondary metabolite productions including phenolic compounds such as lignin in plants (Rozema et al., 1997; Yamasaki et al., 2007). This prompted us to investigate $G m C C R$ RNA expression by UV-B irradiation (Fig. 3C, D). It was shown that $G m C C R$ RNA expression gradually increased and reached the maximum level at $8 \mathrm{~h}$ under UV-B radiation (Fig. 3C). UV-B was irradiated to the etiolated seedlings in either dark or light conditions (Fig. 3D). Without UV-B, GmCCR RNA was not expressed in dark-treated samples, but weakly expressed in light-illuminated samples (Fig. 3D). There was no significant difference in GmCCR RNA expression in the UV-B irradiated seedlings under either dark or visible light conditions (Fig. 3D). Taken together, RNA expression of $G m C C R$ gene is regulated by wounding, drought, high salinity or UV-B stresses in plants. Induction of GmCCR RNA expression may contribute to defense responses to abiotic stresses in plants.

There are accumulating evidences that CCR expression is correlated with lignin accumulation in plants (Jones et al., 2001; Kawasaki et al., 2006; Piquemal et al., 1998; van der Rest et al., 2006). However, it has been suggested that CCR-mediated reaction products monolignols are not only destined for polymerization into lignins but into others such as antioxidants or biocides (Lacombe et al., 1997; Lewis and Davin, 1994). It is not clear if GmCCR might be involved in lignin deposition or in production of polyphenols or antioxidants at this point.

Here, we have shown for the first time, implication of CCR-like protein, GmCCR in defense responses to wounding, drought, high salinity and UV-B stresses.

\section{Acknowledgments}

This research was supported by Basic Science Research Program through the National Research Foundation of Korea (KRF) funded by the Ministry of Education, Science and Technology (KRF-2009-0068370 and -2009-0074413) and by Biogreen 21 (2008-0401034040) funded by the Korean Government.

\section{References}

Boerjan, W., Ralph, J. and Baucher, M. 2003. Lignin biosynthesis. Annu. Rev. Plant Biol. 54:519-546.

Chung, E., Cho, C.-W., Kim, K.-Y., Chung, J., Kim, J.-I., Chung,
Y.-S., Fukui, K. and Lee, J.-H. 2007. Molecular characterization of the GmAMS1 gene encoding $\beta$-amyrin synthase in soybean plants. Russ. J. Plant Physiol. 54:518-523.

Church, G. M. and Gilbert, W. 1984. Genomic sequencing. Proc. Natl. Acad. Sci. USA 81:1991-1995.

Costa, M., Collins, R. E., Anterola, A. M., Cochrane, F. C., Davin, L. B. and Lewis, N. G. 2003. An in silico assessment of gene function and organization of the phenylpropanoid pathway metabolic networks in Arabidopsis thaliana and limitations thereof. Phytochemistry 64:1097-1112.

Jones, L., Ennos, A. R. and Turner, S. R. 2001. Cloning and characterization of irregular xylem4 (irx4): a severely lignin-deficient mutant of Arabidopsis. Plant J. 26:205-216.

Kawasaki, T., Koita, H., Nakatsubo, T., Hasegawa, K., Wakabayashi, K., Takahashi, H., Umemura, K., Umezawa, T. and Shimamoto, K. 2006. Cinnamoyl-CoA reductase, a key enzyme in lignin biosynthesis, is an effector of small GTPase Rac in defense signaling in rice. Proc. Natl. Acad. Sci. USA 103:230235.

Kim, C. Y., Lee, S. H., Park, H. C., Bae, C. G., Cheong, Y. H., Choi, Y. J., Han, C., Lee, S. Y., Lim, C. O. and Cho, M. J. 2000. Mol. Plant-Microbe Interact. 13:470-474.

Klink, V. P., Hosseini, P., Matsye, P., Alkharouf, N. W. and Matthews, B. F. 2009. A gene expression analysis of syncytia laser microdissected from the roots of the Glycine max (soybean) gneotype PI 548402 (Peking) undergoing a resistant after infection by Heterodera glycines (soybean cyst nematode). Plant Mol. Biol. 71:525-567.

Lacombe, E., Hawkins, S., Van Doorsselaere, J., Piquemal, J., Goffner, D., Poeydomenge, O., Boudet, A.-M. and GrimaPettenati, J. 1997. Cinnamoyl CoA reductase, the first committed enzyme of the lignin branch biosynthetic pathway: cloning, expression and phylogenetic relationships. Plant J. 11:429-441.

Lacombe, E., Van Doorsselaere, J., Piquemal, J., Boerjan, W. O., Boudet, A. M. and Grima-Pettenati, J. 2000. Characterization of cis-elements required for vascular expression of the cinnamoyl CoA reductase gene and for protein-DNA complex formation. Plant J. 23:663-676.

Lauvergeat, V., Lacomme, C., Lacombe, E., Lasserre, E., Roby, D. and Grima-Pettenati, J. 2001. Two cinnamoyl-CoA reductase (CCR) genes from Arabidopsis thaliana are differentially expressed during development and in response to infection with pathogenic bacteria. Phytochemistry 57:1187-1195.

Lewis, N. G. and Davin, L. B. 1994. Evolution of lignan and neolignan biochemical pathways. In: Nes D (Ed.), Evolution of Natural Products. ACS Symposium Series American Chemical Society, Washington DC. 562:202-246.

Piquemal, J., Lapierre, C., Myton, K., O’Connell, A., Schuch, W., Gima-Pettenati, J. and Boudet, A. M. 1998. Downregulation of cinnamoylCoA reductase induces significant changes of lignin profiles in transgenic tobacco plants. Plant J. 13:71-83.

Rozema, J., van de Staaij, J., Björn, L. O. and Caldwell, M. 1997. UV-B as an environmental factor in plant life: stress and regulation. Tree 12:22-28.

Shure, M., Wessler, S. and Fedoroff, N. 1983. Molecular identifi- 
cation and isolation of Waxy locus in maize. Cell 35:225-233.

Vance, C. P., Kirk, T. K. and Sherwood, R. T. 1980. Lignification as a mechanism of disease resistance. Annu. Rev. Phytopathol. 18:259-288

Van der Rest, B., Danoun, S., Boudet, A.-M. and Rochange, S. F. 2006. Down-regulation of cinnamoyl-CoA reductase in tomato (Solanum lycopersicum L.) induces dramatic changes in soluble phenolic pools. J. Exp. Bot. 57:1399-1411.

Yamasaki, S., Noguchi, N. and Mimaki, K. 2007. Continuous UV$\mathrm{B}$ irradiation induces morphological changes and the accumulation of polyphenolic compounds on the surface of cucumber cotyledons. J. Radiat. Res. 48:443-454. 